\title{
Comparing Open Preperitoneal Versus Laparoscopic Totally Extra Peritoneal Repair of Inguinal Hernia
}

\author{
E.M.Abdelhafez, H.G.Elgohary, H.E.Ali and A.M.A.M.Ghalab \\ General surgery, Dept., Faculty of Medicine, Benha Univ., Benha, Egypt
}

\section{Abstract}

E-mail:

\begin{abstract}
Background: Hernia repair is one of the most frequent general surgeons operations. The objective of the research is to compare the outcomes of the laparoscopic TEP hernia complete repair with mesh with the results of the open preperitoneal repair with mesh. Methods: There were 60 patients split into two groups, 30 for each. Group O; was subject to preperitoneal mesh repair open, Group L; was subject to mesh laparoscopic TEP repair. In the outpatient clinic at Benha University Hospitals patient follow-up was conducted 7 days after discharge and at 1, 3, 6 and 12 months following surgery until December 2020. The operating method, surgery, post-operational problems, early post-surgical discomfort within one week, hospital stay, physical activity restrictions and incidence of recurrence and chronic pain were also compared. Results: TEP repair was more challenging technically, as indicated by higher operating time, conversion and intraoperative problems. It requires a lengthy learning curve and a committed technical excellence team. However, it is preferable since it is linked with less initial postoperative discomfort, fewer problems related to wounds, shorter hospital stay and quick return to regular activities. Good aesthetic results and overall patient satisfaction are also monitored. Conclusion: Both methods are deemed safe and effective at comparable recurrence and chronic pain rates, but more study is needed.
\end{abstract}

Keywords: Preperitoneal, Laparoscopic, Extra Peritoneal, Repair, Inguinal, Hernia.

\section{Introduction}

Hernia is described as an abnormal protrusion by a defect in the surrounding walls of an organ or tissue. Inguinal and ventral hernias of the anterior abdominal wall are included. Hernia repair is one of the most frequent general surgeon operations. No surgeon has perfect outcomes, despite the frequency of this surgery, and problems including postoperative discomfort, nerve damage, infections and recurrence continue[1].

Inguinal hernia repair is the most common hernia surgery done. The conventional technique of correction of inguinal hernia had changed over a hundred years before the synthetic mesh was introduced. This mesh may be put with an open method or with a laparoscopic minimum access procedure. The rate of recurrence between laparoscopic and open mesh hernia repair techniques is not clearly different. It has been discovered that reduced discomfort and numbness are recommended after laparoscopic repair. It is quicker to return to normal activities[2].

Laparoscopic methods are more and more utilised to treat abdominal hernias and provide the potential advantages of minimum access surgery, maybe a reduced recurrence rate and cheaper costs according to a randomised, controlled trial performed by Olmi et al.[3].

They are successful for the overwhelming majority of patients with primary or recurring hernia and result in a low recurrence rate with excellent patient satisfaction ratings. However, operating periods are longer and severe vascular injuries in less experienced surgeons seem to be at increased risk [2].

Therefore, both laparoscopic as well as open methods to hernia should be known to surgeons who repair the abdominal wall defects to provide the patient with the most suitable method of repair based on individual patient variables and hernia defect features [4].

The objective of this research is to compare open preperitoneal technology with laparoscopic method for completely extra peritoneal repair of inguinal hernea and to shed some light on intraoperative problems and on satisfaction and complications for postoperative patients.

\section{Patients and methods}

This is a prospective randomized comparative study between open preperitoneal versus laparoscopic totally extraperitoneal mesh repair of inguinal hernia. The study included 60 adult patients that were presented in the outpatient clinic at Benha University Hospitals at the period between January 2019 and December 2019 and followed up till December 2020. Follow up is designed for 12 months duration.

The clinical diagnosis of inguinal hernia was based on symptoms and signs elicited during clinical assessment.

\subsection{Patients were classified into}

○ Group (O): 30 patients underwent open preperitoneal mesh hernioplasty.

○ Group (L): 30 patients underwent laparoscopic total extraperitoneal (TEP) mesh hernioplasty.

\subsection{The inclusion criteria}

Include all adult patients with inguinal hernias either unilateral or bilateral or recurrent.

\subsection{Exclusion criteria}

○ Patients with strangulated or obstructed hernias.

o Patients with morbid obesity. 
o Patients with history of recent lower abdominal surgery or irradiation or previous preperitoneal surgery.

o Patients with active skin infection.

o Patients with mental disorders.

o Patients with chronic liver disease with ascites.

o Old patients with benign prostatic hypertrophy and having obstructive urinary symptoms.

$\circ$ Patients with score $\geqq 3$ on American Society of Anesthesiologists (ASA) scale.

\subsection{Preoperative assessment:}

- Clinical history

- Clinical examination

- Routine preoperative work up including:

o Pelvi abdominal ultrosonography and inguino scrotal ultrasonography.

o Complete bloo dcount.

- Liver and kidney functions tests.

- Coagulation profile.

o Random blood sugar

○ ECG, chest X-ray and echocardiography when needed.

\subsection{Preoperative Preparation}

o All the patients were asked to fast for 6 hours preoperatively.

o Patients were asked to micturate before surgery. A urinary catheter was not routinely inserted before either procedure.

o Abdominal and groin hair was shaved from costal margin till midthigh at operating theatre.

o All cases received general anaesthesia.

o Prophylactic antibiotic in the form of $1.2 \mathrm{gm}$ Amoxicillin Clavulanate (Augmentin) injection was given at induction of anesthesia.

○ The operations will be performed by staff surgeons using the same technique and rules.

\subsection{Operative Techniques}

A) Open preperitoneal repair with mesh

B) Laparoscopic TEP repair with mesh.

\subsection{Data recorded}

o The duration of operation in minutes (skin to skin).

o Type of hernia according to the Nyhus classification.

○ Intraoperative complications.

\subsection{Postoperative management}

$\circ$ Patients were assessed regularly during hospital stay.

o Postoperative analgesia was received as Diclofenac sodium (voltaren) $75 \mathrm{mg}$ IM. / 12 hours for one day. Then, Declofenac sodium (voltaren) $50 \mathrm{mg}$ tablets were given on demand later on.

o The postoperative pain assessment was done in the first postoperative day six hours after last analgesic dose administration at rest. Patients were asked to describe their pain levels and the five points verbal rating pain scores (VRS) was documented as follows: $0=$ no pain, $1=$ mild pain, $2=$ moderate pain, $3=$ severe pain and $4=$ unbearable pain) (Loos et al., 2007).

o Patients were asked to report their total ondemand intake of oral analgesics during the first week after surgery and to stop analgesia six hours before coming to the clinic and also were asked to bring with them the medicines strips.

$\circ$ Before discharge, all patients received the same postoperative instructions (limitation on heavy weight lifting for 4 weeks) and were encouraged to return to normal activities as soon as possible.

o Patients were discharged if pain is adequately controlled and free of significant complications.

\subsection{Data recorded}

o Early postoperative complications e.g.: urine retention, wound or scrotal haematoma, etc.

o Hospital-stay in days from day of operation till day of discharge.

o Verbal rating pain score at first postoperative day (VRS1).

\subsection{Statistical analysis}

The gathered data was reviewed, coded, tabulated and entered into a PC using the social science statistical package (SPSS 15.0.1 for windows; SPSS Inc, Chicago, IL, 2001). The data was provided and appropriate analyses were performed based on the kind of data for each parameter collected. Statistics descriptive: Mean default deviation $( \pm$ SD), median and numerical data range. Non-numerical data frequency and proportion. Statistics analytical: The student Test was used to evaluate the statistical significance of the difference between the two research group meanings. In order to evaluate the statistical significance of the difference of a nonparametric variable between two research groups, the Mann Whitney test (U test) was employed. The Chi-Square test was used to investigate the connection between two qualitative variables. Exact test from Fisher: the connection between two qualitative variables was examined when the anticipated count is less than 5 in more than $20 \%$ of cells. Analysis of correlation (using the Pearson method): To evaluate the association strength between two quantitative variables. The symbolically described correlation coefficient " $\mathrm{r}$ " indicates the degree and direction of the linear connection between two variables. P- value: significance level: $\mathrm{P}>0.05$ : Non-important $(\mathrm{NS}) . \mathrm{P}<$ 0.05: Meaningful (S).

\section{Results}

The study was performed at Benha university hospitals, and included 60 patients all of them were adult males reflecting the great sex predilection of this disease. The age of the study group ranged between 
20 and 65 years with a mean of $42.5 \pm 12.3$ years. The study group's BMI ranged between 18 and 32 with a mean of $24.9 \pm 2.45 \mathrm{Kg} / \mathrm{m} 2$. Sixty patients were included in this study, all of them were males. Their age ranged between 20 and 65 years with a mean and standard deviation (SD) of $42.5 \pm 12.3$ years.

Group (O): 28 patients $(93.3 \%)$ had unilateral inguinal hernia, while 2 cases $(6.6 \%)$ had bilateral oblique inguinal hernia. So we had 30 patients with 32 hernias. 27 patients $(90 \%)$ had primary hernias, while $3(10 \%)$ had recurrent hernias after anterior repair. $71.8 \%(n=23)$ of hernias were oblique inguinal hernias, $18.7 \%(\mathrm{n}=6)$ of hernias were direct inguinal hernias and $9.3 \%(\mathrm{n}=3)$ were dual hernias. Group (L): 26 cases $(86.6 \%)$ had unilateral inguinal hernia, while 4 cases $(13.3 \%)$ had bilateral inguinal hernia, two of them were oblique. So we had 30 patients with 34 hernias. 27 patients $(90 \%)$ were primary, while 3 hernias $(10 \%)$ were recurrent. $73.5 \%(\mathrm{n}=25)$ of hernias were oblique inguinal hernias, $20.5 \%(\mathrm{n}=7)$ of hernias were direct inguinal hernias and $5.8 \%(\mathrm{n}=$ 2 ) were dual hernias. Fig. $(1,2)$

Comparison between both study groups as regards operative time in table (1)

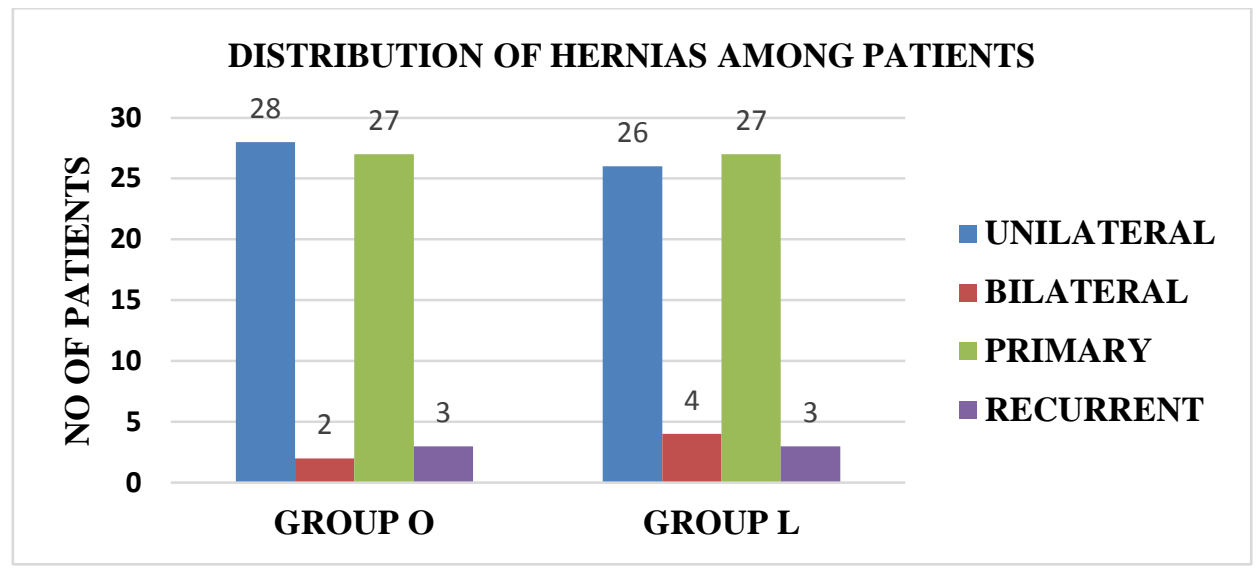

Fig. (1) General hernia distribution among patients of the study group.

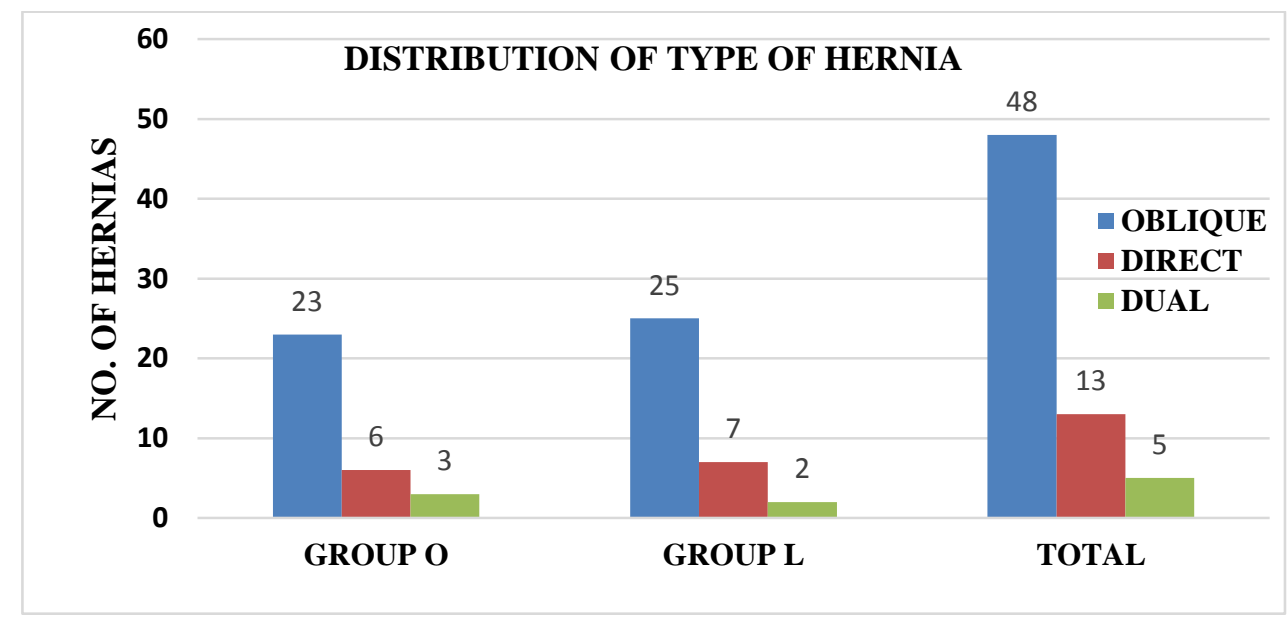

Fig. (2) Distribution of type of hernias in the study group.

Table (1) Description and Comparison between both study groups as regards operative time

\begin{tabular}{|c|c|c|c|c|c|c|c|c|}
\hline \multirow{3}{*}{ Operative time } & \multicolumn{6}{|c|}{ Group } & \multirow{3}{*}{$P^{*}$} & \multirow{3}{*}{ Sig } \\
\hline & \multicolumn{3}{|c|}{ Group O } & \multicolumn{3}{|c|}{ Group L } & & \\
\hline & Mean & \pm SD & Median & Mean & \pm SD & Median & & \\
\hline Total & 65.2 & 19.7 & 60.0 & 83.8 & 19.8 & 85.0 & $.001 *$ & HS \\
\hline unilateral cases & 59.80 & 14.47 & 60.00 & 78.91 & 13.48 & 85.00 & $.049 *$ & $\mathrm{~S}$ \\
\hline bilateral cases & 95.00 & 21.21 & 95.00 & 100.00 & 37.42 & 95.00 & $.874 *$ & NS \\
\hline recurrent cases & 90.00 & 26.45 & 91.00 & 100.00 & 20 & 95.00 & $.629 *$ & NS \\
\hline
\end{tabular}

* Student $t$ test 
Intraoperative complications in table (2)

There was no statistically significant difference between Group $\mathrm{O}$ and $\mathrm{L}$ cases as regard postoperative complications. However, wound-related complications occurred exclusively in group O. Early postoperative complications are shown in table (3)

There was a significant difference between Group $\mathrm{O}$ and $\mathrm{L}$ cases as regard VRS at 1st postoperative day, as group $\mathrm{O}$ cases showed higher mean VRS compared to group L cases (1.73 vs 1.27). This was also evident in the unilateral and bilateral subgroups. The recurrent cases showed no significant difference in pain scores between both groups. table (4)

No significant difference was detected between Group $\mathrm{O}$ and $\mathrm{L}$ cases as regards functional limitation score. However, there was significant difference in subgroup of bilateral patients favoring group L. table (5)

Table (2) Intraoperative complications in Group O and L.

\begin{tabular}{|c|c|c|c|c|c|c|c|}
\hline \multirow{3}{*}{ Intraoperative complications } & & \multicolumn{4}{|c|}{ Group } & \multirow{3}{*}{$\mathbf{P *}$} & \multirow{3}{*}{ Sig } \\
\hline & & \multicolumn{2}{|c|}{ Group 0} & \multicolumn{2}{|c|}{ Group L } & & \\
\hline & & $\mathbf{N}$ & $\%$ & $\mathbf{N}$ & $\%$ & & \\
\hline \multirow{2}{*}{ Peritoneal tears } & Yes & 4 & $13.3 \%$ & 4 & $13.3 \%$ & \multirow{2}{*}{$1.0 * *$} & \multirow{2}{*}{ NS } \\
\hline & No & 26 & $86.7 \%$ & 26 & $86.7 \%$ & & \\
\hline \multirow{2}{*}{ Bleeding } & Yes & 0 & $.0 \%$ & 2 & $6.7 \%$ & \multirow{2}{*}{$0.492 * *$} & \multirow{2}{*}{ NS } \\
\hline & No & 30 & $100.0 \%$ & 28 & $93.3 \%$ & & \\
\hline Total & & 4 & $13.3 \%$ & 6 & $20 \%$ & & \\
\hline
\end{tabular}

Table (3) Early postoperative complications in Group O and Group L.

\begin{tabular}{|c|c|c|c|c|c|c|c|}
\hline \multirow{3}{*}{ postoperative complication } & & \multicolumn{4}{|c|}{ Group } & \multirow{3}{*}{$\mathbf{P}^{*}$} & \multirow{3}{*}{ Sig } \\
\hline & & \multicolumn{2}{|c|}{ Group O } & \multicolumn{2}{|c|}{ Group L } & & \\
\hline & & $\mathbf{N}$ & $\%$ & $\mathbf{N}$ & $\%$ & & \\
\hline \multirow[b]{2}{*}{ Urine retention } & Yes & 2 & $6.7 \%$ & 1 & $3.3 \%$ & \multirow{2}{*}{$1.0^{* *}$} & \multirow[b]{2}{*}{ NS } \\
\hline & No & 28 & $93.3 \%$ & 29 & $96.7 \%$ & & \\
\hline \multirow{2}{*}{ Testicular discomfort } & Yes & 1 & $3.3 \%$ & 1 & $3.3 \%$ & \multirow{2}{*}{$1.0^{* *}$} & \multirow{2}{*}{ NS } \\
\hline & No & 29 & $97.7 \%$ & 29 & $97.7 \%$ & & \\
\hline \multirow{2}{*}{ Inguinoscrotal hematoma } & Yes & 3 & $10.0 \%$ & 4 & $13.3 \%$ & \multirow{2}{*}{$1.0^{* *}$} & \multirow{2}{*}{ NS } \\
\hline & No & 27 & $90.0 \%$ & 26 & $86.7 \%$ & & \\
\hline \multirow[b]{2}{*}{ inguinoscrotal seroma } & Yes & 5 & $16.7 \%$ & 3 & $10.0 \%$ & \multirow[b]{2}{*}{$.706^{* *}$} & \multirow[b]{2}{*}{ NS } \\
\hline & No & 25 & $83.3 \%$ & 27 & $90.0 \%$ & & \\
\hline \multirow{2}{*}{ Wound hematoma } & Yes & 1 & $3.3 \%$ & 0 & $.0 \%$ & \multirow{2}{*}{$1.0^{* *}$} & \multirow{2}{*}{ NS } \\
\hline & No & 29 & $96.7 \%$ & 30 & $100.0 \%$ & & \\
\hline \multirow{2}{*}{ Wound seroma } & Yes & 2 & $6.7 \%$ & 0 & $.0 \%$ & \multirow{2}{*}{$.492 * *$} & \multirow{2}{*}{ NS } \\
\hline & No & 28 & $93.3 \%$ & 30 & $100.0 \%$ & & \\
\hline \multirow{2}{*}{ Wound infection } & Yes & 2 & $6.7 \%$ & 0 & $.0 \%$ & \multirow{3}{*}{$.492 * *$} & \multirow{3}{*}{ NS } \\
\hline & No & 28 & $93.3 \%$ & 30 & $100.0 \%$ & & \\
\hline total & & 16 & $53.3 \%$ & 9 & $30 \%$ & & \\
\hline
\end{tabular}

Table (4) Description and Comparison between both study groups as regards VRS at $1^{\text {st }}$ postoperative day.

\begin{tabular}{|c|c|c|c|c|c|c|c|c|}
\hline \multirow{3}{*}{$\begin{array}{l}\text { VRS at } 1^{\text {st }} \text { postoperative day } \\
\text { (VRS1) }\end{array}$} & \multicolumn{6}{|c|}{ Group } & \multirow{3}{*}{$\mathbf{P}^{*}$} & \multirow{3}{*}{ Sig } \\
\hline & \multicolumn{3}{|c|}{ Group O } & \multicolumn{3}{|c|}{ Group L } & & \\
\hline & Mean & $\pm \mathrm{SD}$ & Median & Mean & \pm SD & Median & & \\
\hline Total & 1.73 & .74 & 2 & 1.27 & .78 & 1 & $.021 *$ & $\mathrm{~S}$ \\
\hline Unilateral cases & 1.52 & .59 & 2.00 & 1.00 & .60 & 1.00 & $.004 *$ & HS \\
\hline Bilateral cases & 3.00 & .00 & 3 & 1.2 & .82 & 2 & $.021 *$ & $\mathrm{~S}$ \\
\hline Recurrent cases & 2.67 & .58 & 3 & 2.33 & .58 & 2 & $.456 *$ & NS \\
\hline
\end{tabular}

No significant difference was detected between Group O and L cases as regards hospital stay. Fig. (3) 


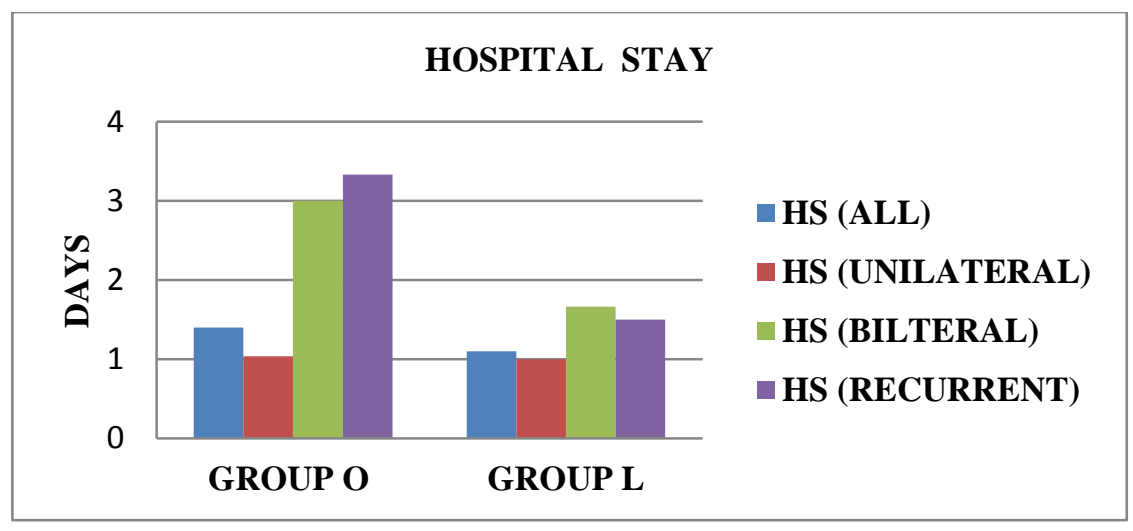

Fig. (3) Postoperative Hospital stay $(H S)$ in group O and L.

Table (5) Description and Comparison between both study groups as regards functional limitation score at $7^{\text {th }}$ postoperative day (FLS7).

\begin{tabular}{|c|c|c|c|c|c|c|c|c|}
\hline \multirow{3}{*}{ Functional limitation score } & \multicolumn{6}{|c|}{ Group } & \multirow{3}{*}{$\mathbf{P *}$} & \multirow{3}{*}{ Sig } \\
\hline & \multicolumn{3}{|c|}{ Group O } & \multicolumn{3}{|c|}{ Group L } & & \\
\hline & Mean & \pm SD & Median & Mean & \pm SD & Median & & \\
\hline Total & 3.93 & 1.26 & 3 & 3.50 & .78 & 3 & $.114 *$ & NS \\
\hline Unilateral cases & 3.48 & .71 & 3.00 & 3.22 & .42 & 3.00 & $.132 *$ & NS \\
\hline Bilateral cases & 6.50 & .71 & 7 & 4.25 & .96 & 5 & $.045 *$ & $\mathrm{~S}$ \\
\hline Recurrent cases & 6.00 & 1.00 & 6 & 4.67 & 1.15 & 4 & $.205^{*}$ & NS \\
\hline
\end{tabular}

None of our patients can be tagged as having chronic pain. Nevertheless, we had two patients of group $(\mathrm{O})$ suffering from non specific groin pain and discomfort with occasional parathesia around the incision line and foreign body sensation in the groin. At the three months postoperative visit, all patients of both groups returned to their usual ordinary activity. There was no case of recurrence in either group during the follow-up period of 12 months.

\section{Discussion}

26/60 (43.3\%) of patients suffered from postoperative difficulties in our study. All our postoperative issues have been handled and endured effectively by our patients. A second operation was not necessary since most of the issues were handled conservatively in order to reflect the overall safety of the surgery.

In group $\mathrm{O}$ and $\mathrm{L}, 53.3$ percent compared to 30 percent problems were observed. This clearly favours the TEP group with far less post-operational issues than the open group.

The most common post-operative complication in GroupO and L were hematoma and/or seroma (including Hematocele and Hydrocele) that affected $15 / 60$ (25\%), which had nearly similar distribution across both study groups, respectively (8/30:26.6\% vs $7 / 30: 23.3 \%$ ). This was mostly done in those who had long hernial bags, especially inguinal hernia. This was also evident in individuals with large straight hernias and bilateral hernias. Chronic liver disease patients were also affected.
Our approach is to limit these people hernially detached from the cord by connecting and transecting the hernia and reducing the proximal section while leaving the distal portion accessible. Blood and fluid collected at the distal end. Without operative drainage and haematoma the patients were treated with canned products and gradually reduced in size after one month till resolution. Moreover, the Stoppa idea of inhibiting the repair of the groyne muscles is in line with our approach. However, the cost of leaving a little wall bulge seems to collect post-operative fluids.

Another explanation could be because establishing a reasonably broad range of operations involves cutting more lymph nodes and reducing corporal fluid drainage. The excess transversal fascia may be picked up at its bulging, invaginated apex and brought to the front abdominal wall and the Coop ligament to minimise dead areas with large direct sacks. The usage of the redundant TF may also be tried to place the PDS endoloop on its base[5]. On the contrary, some surgeons believed it was not necessary, and the dead area closed spontaneously after absorption of fluid[6].

Recurring hernia was similar to inguinoscrotal haematoma and two patients required ultrasound to exclude recurrence. Postoperative pressure and scrotal support were provided by " $\alpha$-chemotrypsin" injection for three days followed by "Alphentern" for one week. The cornerstone of treatment was reassurance and cautious expectation. Literature shows that haematoma was occasionally required for aspirations or surgical evacuations, but not in our instance. 
However, in late cases of our study, we started to drain these dangerous individuals with acceptable results.

In contrast to inguinoscrotal seroma/hematoma, wound hematoma and seroma in group $\mathrm{O}(3 / 30$ or 10 percent) are more common than zero in TEP.

The frequency of superficial wound infection was 3.3 percent (2/60). Despite the usual preventative antibiotic dose provided to patients in both groups, it was detected in $2 / 30$ Group $O$ patients. One was diabetic, the other COPD-positive and had bilateral inguinal hernia.

According to a recent analysis by Cochrane of 17 studies assessing prophylactic antibiotic usage, total infection levels were $3.1 \%$ and $4.5 \%$ [7].

None of our patients had mesh-related profound infections. Overall, the deep groyne infection incidence is rare and $0.3 \%-0.6 \%[8]$. The deepening of the mesh in the preperitoneal technique may support this further.

Urine retention was reported as most studies (4-8 percent) in 3 ( 5 percent), 2 in Group $\mathrm{O}$ and 1 in Group $\mathrm{L}[9]$. This may be attributed to the age ( $>55$ years) with a prostatic growth history of these persons. All were treated conservatively without catheterization.

We have not routinely introduced urine catheter into our study and have requested our subjects to urinate before surgery. However, if the surgery duration is long and the patient gets a large amount of $\mathrm{IV}$, the anesthesiologist sometimes ordered a table catheterization. Nelaton catheter is used and withdrawn before patient recovery.

Testicular discomfort occurred in $2 / 60$ people (3.3\%) and was equally distributed across both $\mathrm{O}$ and L groups. In the first two days after surgery, many people experienced unexplained testicular discomfort or agony. A patient with a duplex examination was somewhat enlarged and sensitive to testicular blood disturbances. Both persons were afebrile with normal leucocyte numbers. The illness was spontaneously healed in both instances within 4-7 days after surgery.

Test-related problems occur from $0.9 \%$ to $9 \%$ of all repairs of the inguinal hernia[10]. There is also pain, orchitis and atrophy. The genesis of pain is unknown and is thought to result in injury to the genitophemoral nerve or plexus. Testicular venous thrombosis is not an obstacle to artery fluid due to the extensive collateral flow between the vesic, prostatic, testicular and deferential artery branches. Orchitis postoperative Indoors and outdoors, the scrotal branches of the pudendal arteries likewise anastomize with the sperm cord vessels. The deferential artery is believed to provide the testis with sufficient blood supply [11].

Postoperative hospital stays were also longer in Group $\mathrm{O}$ than TEP durations (1.4 vs. 1.1 days). In many publications sources, the laparoscopic method has demonstrated a small decrease in time, although this is not universal [12].
The literature has a very large number of studies that report the length of hospital stay with quite different variances. Although a significant predictor of the early post-operative results and expenses rather than the technology influences the duration of hospital stays substantially more than the hospital policy. The average length of stay was greater in different hospitals than in different operative techniques [2].

Our results are consistent with several research studies comparing laparoscopic and open groups with discomfort and physical activity $[2,13]$.

\section{Conclusion}

The technically difficult TEP repair in our study appeared to be shown by increased operating time, conversion and intra-working issues. Both operations are considered safe since patients have a good tolerance of all perioperative issues and there is no need for a second surgery. The early postoperative issues with higher wound complications in the open group showed similar results in both approaches. TEP repair patients had less immediate post-operative discomfort than pain reduction and painkiller consumption when compared with open repair. TEP repair is associated with shorter hospital stays and faster return to normal operations. Our findings showed a positive trend for TEP repair compared to an open group in bilateral and recurrent instances. More concentrated research is required, though. Both techniques succeed in the treatment of inguinal hernia and are connected to 0 percent recurrence. Due to the small sample size and short follow-up time, long-term study is recommended.

\section{References}

[1] C. M. Townsend, R. D. Beauchamp, B. M. Evers, and K. L. Mattox, Sabiston textbook of surgery E-book. Elsevier Health Sciences, vol. 10, pp. 43-51, 2016.

[2] K. McCormack, B. L. Wake, C. Fraser, L. Vale, J. Perez, and A. Grant, "Transabdominal pre-peritoneal (TAPP) versus totally extraperitoneal (TEP) laparoscopic techniques for inguinal hernia repair: a systematic review," Hernia, vol. 9, pp. 109-114, 2005.

[3] S. Olmi, A. Scaini, G. C. Cesana, L. Erba, and E. Croce, "Laparoscopic versus open incisional hernia repair," Surg. Endosc., vol. 21, pp. 555559, 2007.

[4] J. Ruiz, A. Barrios, A. Lora, V. Vega, G. Florez, and F. Mendivelso, "Extraperitoneal laparoscopic ventral hernia repair: one step beyond," Hernia, vol. 23, pp. 909-914, 2019.

[5] S. Putnis and C. R. Berney, "Totally extraperitoneal repair of inguinal hernia: techniques and pitfalls of a challenging procedure," Langenbeck's Arch. Surg., vol. 397, pp. 1343-1351, 2012.

[6] Voeller, "Challenging Hernias Postgraduate," vol. 2008, pp. 65-78, 2008. 
[7] F. J. Sanchez-Manuel, J. Lozano-García, and J. L. Seco-Gil, "Antibiotic prophylaxis for hernia repair.," Cochrane Database Syst. Rev., vol. 2, pp. CD003769, 2012.

[8] T. J. Aufenacker, M. J. W. Koelemay, D. J. Gouma, and M. P. Simons, "Systematic review and meta-analysis of the effectiveness of antibiotic prophylaxis in prevention of wound infection after mesh repair of abdominal wall hernia," J. Br. Surg., vol. 93, pp. 5-10, 2006.

[9] M. V Sivasankaran, T. Pham, and C. M. Divino, "Incidence and risk factors for urinary retention following laparoscopic inguinal hernia repair,” Am. J. Surg., vol. 207, pp. 288292, 2014.

[10] S. W. Nienhuijs, C. Rosman, L. J. a Strobbe, a Wolff, and R. P. Bleichrodt, "An overview of the features influencing pain after inguinal hernia repair.," Int. J. Surg., vol. 6, pp. 351-6, 2008
[11] M. S. L. Liem, E. B. van Duyn, Y. van der Graaf, and T. J. M. V van Vroonhoven, "Recurrences after conventional anterior and laparoscopic inguinal hernia repair: a randomized comparison.," Ann. Surg., vol. 237, pp. 136-41, 2003.

[12]F. Wittenbecher, D. Scheller-Kreinsen, J. Röttger, and R. Busse, "Comparison of hospital costs and length of stay associated with openmesh, totally extraperitoneal inguinal hernia repair, and transabdominal preperitoneal inguinal hernia repair: an analysis of observational data using propensity score matching," Surg. Endosc., vol. 27, pp. 13261333, 2013.

[13] E. Kuhry, R. N. Van Veen, H. R. Langeveld, E. W. Steyerberg, J. Jeekel, and H. J. Bonjer, "Open or endoscopic total extraperitoneal inguinal hernia repair? A systematic review," Surg. Endosc., vol. 21, pp. 161-166, 2007. 